\title{
WD-repeat instability and diversification of the Podospora anserina hnwd non-self recognition gene family
}

\author{
Damien Chevanne, Sven J Saupe, Corinne Clavé and Mathieu Paoletti*
}

\begin{abstract}
Background: Genes involved in non-self recognition and host defence are typically capable of rapid diversification and exploit specialized genetic mechanism to that end. Fungi display a non-self recognition phenomenon termed heterokaryon incompatibility that operates when cells of unlike genotype fuse and leads to the cell death of the fusion cell. In the fungus Podospora anserina, three genes controlling this allorecognition process het-d, het-e and het-r are paralogs belonging to the same hnwd gene family. HNWD proteins are STAND proteins (signal transduction NTPase with multiple domains) that display a WD-repeat domain controlling recognition specificity. Based on genomic sequence analysis of different $P$. anserina isolates, it was established that repeat regions of all members of the gene family are extremely polymorphic and undergoing concerted evolution arguing for frequent recombination within and between family members.

Results: Herein, we directly analyzed the genetic instability and diversification of this allorecognition gene family. We have constituted a collection of 143 spontaneous mutants of the het-R (HNWD2) and het- $E$ ( $h n w d 5)$ genes with altered recognition specificities. The vast majority of the mutants present rearrangements in the repeat arrays with deletions, duplications and other modifications as well as creation of novel repeat unit variants.
\end{abstract}

Conclusions: We investigate the extreme genetic instability of these genes and provide a direct illustration of the diversification strategy of this eukaryotic allorecognition gene family.

\section{Background}

All living organisms have developed processes to discriminate self from non-self. Non-self recognition is required both in conspecific allorecognition (recognition of a different individual from the same species) or recognition of pathogenic non-self. In eukaryotes non-self recognition relies on the production of polymorphic proteins able to react to a range of molecules. Typically, genes involved in non-self recognition are among the most polymorphic loci in eukaryotic genomes, a classical example being the MHC/HLA loci in mammals [1]. Diversification of the corresponding genes occurs despite the exceedingly low overall mutation rate required to maintain genome integrity [2]. A challenge in evolutionary biology is to under-

\footnotetext{
*Correspondence: mathieu.paoletti@ibgc.cnrs.fr

1 Laboratoire de Génétique Moléculaire des Champignons, IBGC, UMR 5095 Université Victor Segalen Bordeaux2 et CNRS, 1 rue Camille Saint-Saëns,33077 Bordeaux Cedex, France

Full list of author information is available at the end of the article
}

stand the mechanisms explaining this high level of polymorphism in non-self recognition genes. Clearly, positive selection for beneficial variants participates in the maintenance of polymorphism in non-self recognition genes, but in addition in a number of cases specific molecular mechanisms that increase diversification (and thus increase the panel of alleles on which selection can act on) are also involved.

In filamentous fungi conspecific non-self recognition happens in the heterokaryotic cell produced after the somatic fusion by anastomosis of cells of unlike genotypes [3-5]. The vegetative incompatibility (VI) process takes place when incompatible alleles of specific het loci are co-expressed in the cytoplasm of the heterokaryon and leads to a cell death reaction [6]. The biological function of VI and forces driving the evolution and maintenance of het genes remain unclear. VI is thought to restrict transmission of deleterious cytoplasmic elements such as viruses or abnormal mitochondria [7,8], and to 
limit resource plundering by aggressive genotypes [9]. It has also been proposed that genes controlling VI could in fact be involved in pathogen recognition and that VI could be a secondary consequence of the pathogendriven divergence in these genes $[10,11]$. In that hypothesis, VI would be analogous to a genetic incompatibility phenomenon common in plants and known as hybrid necrosis. In hybrid necrosis a post-zygotic incompatibility occurs in F1 hybrids between different isolates and results from pathogen-driven divergence in genes with an immune function $[12,13]$.

So far het loci have been characterized in only two species, Podospora anserina and Neurospora crassa. In P. anserina the het-d and het-e loci are involved in nonallelic incompatibility interactions with het-c (unrelated to N. crassa het-c) [14] while the het-r locus is involved in non-allelic incompatibility with het-v $[15,16]$ (figure 1). Analysis of the recently sequenced $P$. anserina genome
[17] revealed that het-d, het-e and het-r belong to the hnwd gene family that includes 2 additional members (hnwd1 and hnwd3). They are part of the larger $n w d$ family that comprises five additional members [18]. hnwd genes encode for proteins including a N-terminal HET domain effector of cell death [19-21], a central NACHT domain [22] binding GTP and essential for activity [23], and a C-terminal WD repeat domain that varies in size (number of repeats) and sequence between natural isolates and defines allele specificity [24,25] (Figure 1). The five $h n w d$ genes encode for proteins of the STAND class thought to act as molecular switches with a closed inactive form and an open active form adopted upon binding to an activating molecule $[26,27]$. The $n w d$ genes display the NACHT and WD domains but lack the HET domain.

The WD repeat domains of the P. anserina nwd genes display very dynamic evolutionary features [18]. The WD domain is constituted of a variable number of highly con-

\section{A/}

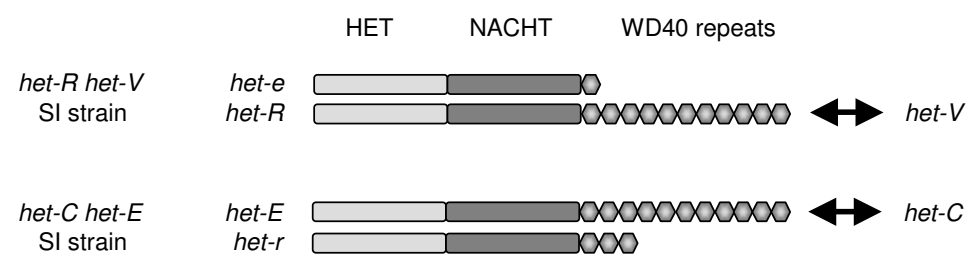

B/

WD40 repeat instability

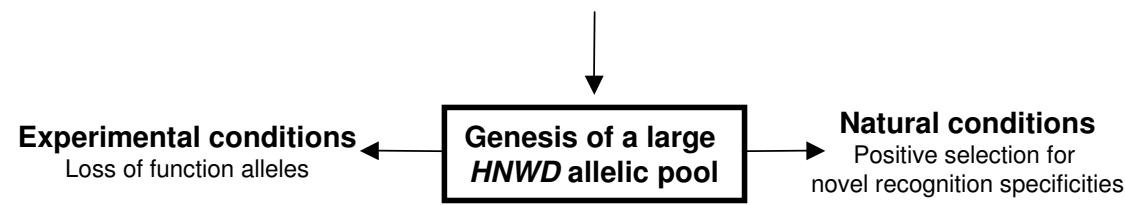

$\mathrm{C} /$
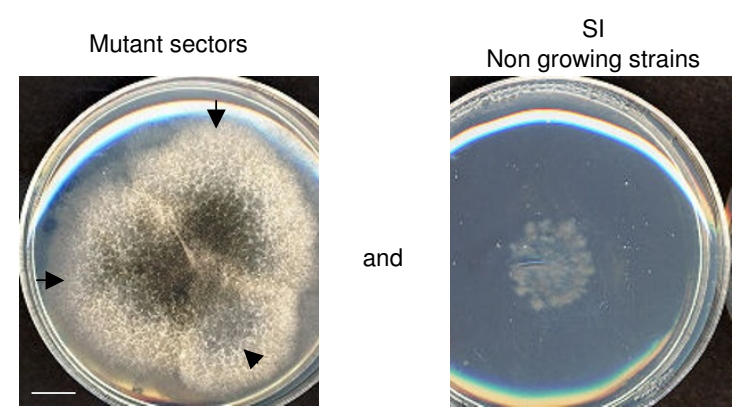

Figure 1 Constitution of the RV collection of mutants. A/Schematic representation of het-e and het-r alleles at the hnwd loci under study in the het- $R$ het $-V$ and the het-C het- $E$ self incompatible strains. Plain arrowheads indicate non allelic incompatible interactions. B/Genesis and selection of new alleles at $h n w d$ loci. During vegetative growth, the WD40 repeat instability promotes the rapid genesis of allelic variants at the $h n w d$ loci for selection to act on. Our experimental set up selects for loss of function mutations while in natural conditions in the wild, selection will promote the maintenance of new recognition specificities. C/Selection of loss of function mutants in our experimental set up. Basically, SI strains were grown for $24 \mathrm{~h}$ in permissive condition before incubation for five days in non permissive conditions. Arrows indicate mutant sectors escaping from cell death from a single culture, as opposed to a culture where no mutant sector appeared. A single mutant per subculture was picked up for further analysis. 
served WD40 repeat units that are submitted to three evolutionary forces: 1) a high mutation load due to the high number of repeats and the possible action of RIP, a mutagenic process targeting repeated sequences [28], 2) concerted evolution resulting from WD40 unit sequence exchanges between and within loci and leading to WD40 repeat unit sequence homogenization, 3) strong positive diversifying selection acting on four positions encoding for amino acids located at the protein-protein interaction surface of the $\beta$ propeller fold adopted by the WD repeat domains [18]. The combination of these evolutionary forces has the potential to constantly generate new alleles. This dynamism does not make obvious sense in the context of a role of these het genes restricted to VI. In contrast, in the context of the hnwd genes encoding for pathogen receptors contributing to an immune response $[29,30]$, rapidly generating new alleles would allow for the surveillance of a wide range of pathogens.

It is well established that tandemly repeated sequences are unstable and undergo repeat number expansions and contractions. This repeat instability is at the origin of numerous cancers and neurological and developmental disorders in humans but in a number of cases this plasticity also appears advantageous as it can favour rapid genome evolution and adaptation [31,32]. Repeat variation rates in micro and minisatellite loci can be 5-orders of magnitude higher than rates of single-nucleotide substitution. Intragenic tandem repeat instability was for instance found to allow rapid evolution of cell surface antigens in fungi [33,34] but also of genes controlling morphological features in domestic dogs [35]. Mechanisms for this instability have been extensively studied and it turns out that any mechanism involving new DNA synthesis such as replication, recombination and repair can contribute to repeat rearrangements [32].

In the present paper, we took advantage of the genetic tractability of non allelic VI systems of $P$. anserina to directly analyze rearrangements in intragenic tandem repeats in this eukaryotic non-self recognition gene family. We selected a collection of 143 loss of function specificity variants in two members of the hnwd family spontaneously generated under vegetative growth conditions (figure 1), while in nature selection would maintain alleles providing new recognition specificities. We then analyzed the underlying molecular events that occurred. We found that diversification of the WD-repeat domains occurs through intragenic reshuffling in the repeat arrays. Importantly, this reshuffling is accompanied by frequent generation of novel repeat unit sequences. The consequences of this diversification in the context of non self recognition in natural isolates will be discussed. This study provides a direct illustration of the role of tandem repeat instability in evolvability in the context of non-self recognition.

\section{Results}

\section{Mutational bias towards hnwd genes}

In non-allelic incompatibility systems, incompatibility is expressed upon cell fusion between incompatible strains but also in the progeny of sexual crosses involving incompatible parental strains. Incompatible gene pairs (such as het- $R$ het $V$ or het- $C$ het-E) can be associated in a single haploid nucleus by appropriate genetic crosses, leading to the production of so-called self incompatible (SI) strains (Figure 1). It has long been described that spontaneous mutants escaping cell death arise from SI strains [16]. The vast majority of the mutations allowing escape from het- $R$ het- $V$ or het-C het- $E$ incompatibility occur in the het- $R$ or het $E$ genes respectively while inactivation of het- $V$ and het- $C$ is rare. The underlying cause for this differential "mutability" was not understood. We generated two collections of spontaneous mutants allowing escape from het- $R$ het- $V$ or het- $C$ het- $E$ incompatibility, the different steps of the process being presented figure 1 and in Materials and Methods. Basically het-R het-V and het-C het- $E$ SI strains were grown for $24 \mathrm{~h}$ in permissive conditions suppressing the manifestations of VI before transfer to non permissive conditions where the incompatibility reaction proceeds. After 5 days, 299 spontaneous mutant sectors recovering normal growth arose from which we picked and analysed a single mutant per subculture to generate the RV and CE spontaneous mutant collections. The RV and CE collections include respectively 109 and 34 unique individuals.

Mutations allowing for suppression of incompatibility could occur a priori in either one of the het genes composing the gene pair causing the incompatible interaction of the SI strain (het-R het-V and het-C het-E), or in extragenic suppressor which we did not observe. For each collection, we identified the mutated het gene by confronting each mutant against both parents used to set up the original cross. Incompatibility is characterized by the formation of an abnormal contact zone between incompatible strains called the barrage [6]. For each gene pair, the mutations were totally biased towards one of the two het genes. All the mutants of the RV collection were affected at the het-r locus and expressed an inactive het-r allele. As well, all the mutants of the CE collection were affected at the het-e locus and expressed an inactive het-e allele. The combined 143 mutants from both collections are affected in the het genes that belong to the hnwd gene family suggesting a particular genetic instability at the hnwd loci.

\section{Repeat loss is the most common event for hnwd inactivation}

We set out to define the molecular events leading to inactivation of the hnwd genes. The presence of highly conserved tandemly repeated WD40 sequences both in the 
het- $E$ and het- $R$ genes led us to investigate the status of the repeat domains. The number of WD40 repeat units present in the WD repeat domains of the mutated genes (het-r or het-e) was estimated from the size of the PCR amplicon generated using locus specific primers (additional file 1). The initial active het- $R$ allele and the het-E1 allele used here both contain 11 WD40 repeats [15]. On the basis of the number of repeats in the WD domain of the mutants, we defined three classes of mutants, the class of deletion mutants (dWD) that present a reduced number of WD40 repeats compared to the wild type alleles, the class of gain of repeat mutants (gWD) that have a greater number of WD40 repeat units than the wild type allele, and the class of mutants with the same number of WD40 repeats as the initial active allele (snWD). As revealed in figure 2, for both collections most mutants belong to the dWD class as $87 \%(95 / 109)$ of the RV mutants and 79\% (27/34) of the CE mutants have less than the original 11 WD40 repeats. In addition we found
10 snWD class and 4 gWD class mutants in the RV collection, and 3 snWD class and 4 gWD class mutants in the $\mathrm{CE}$ collection. The proportion of dWD mutants was to be expected as it was shown that a minimum of ten WD40 repeat units are required for VI to occur [24].

\section{Mapping of the WD40 repeat deletions}

Most mutants are thus generated through deletion of a number of WD40 repeat sequences. Deletions of a small number of WD40 repeats are more frequent than deletions of a large number of repeats (figure 2). We took advantage of the presence of BglII restriction site polymorphism in the WD40 repeat sequences of the het- $R$ parental allele [15] to map the deletion events of the RV deletion mutants collection (Figure 3). First we predicted all possible patterns generated by $B g l \mathrm{II}$ restriction of PCR fragments amplified with primers A and B from genomic DNA of the het-R wild type allele or all the deletion mutants of the RV collection (additional file 2). Then,

A

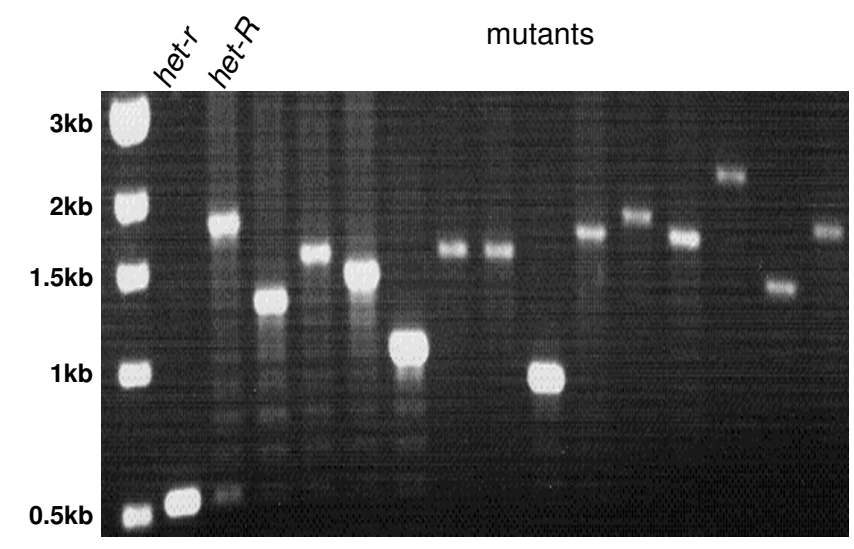

B/

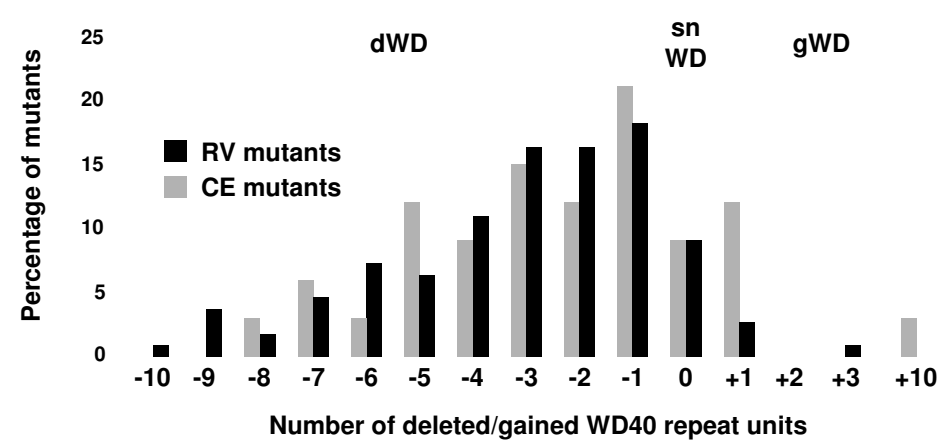

Figure 2 Classes of mutants identified by the number of WD40 repeat units. A/The WD domain of the het-r gene of a selection of mutants from the RV collection were PCR amplified and separated on an 0.8\% agarose gel (molecular marker: $1 \mathrm{~kb}$ ladder). The sizes of the amplicons are compared to the natural inactive (het-r) and active (het- $R$ ) alleles. B/Three classes of mutants are defined according to the size of the WD repeat domain of the het-r or the het-e gene: the deletion mutants (dWD), the gain of WD40 repeat mutants (GWD) and the mutants with the same number of WD40 repeats as the initial allele (snWD). Subclasses of mutants are defined according to the number of WD40 repeat units deleted (-) or gained (+) compared to the initial allele. The percentage of the total number of mutants comprised in each subclass is represented. 


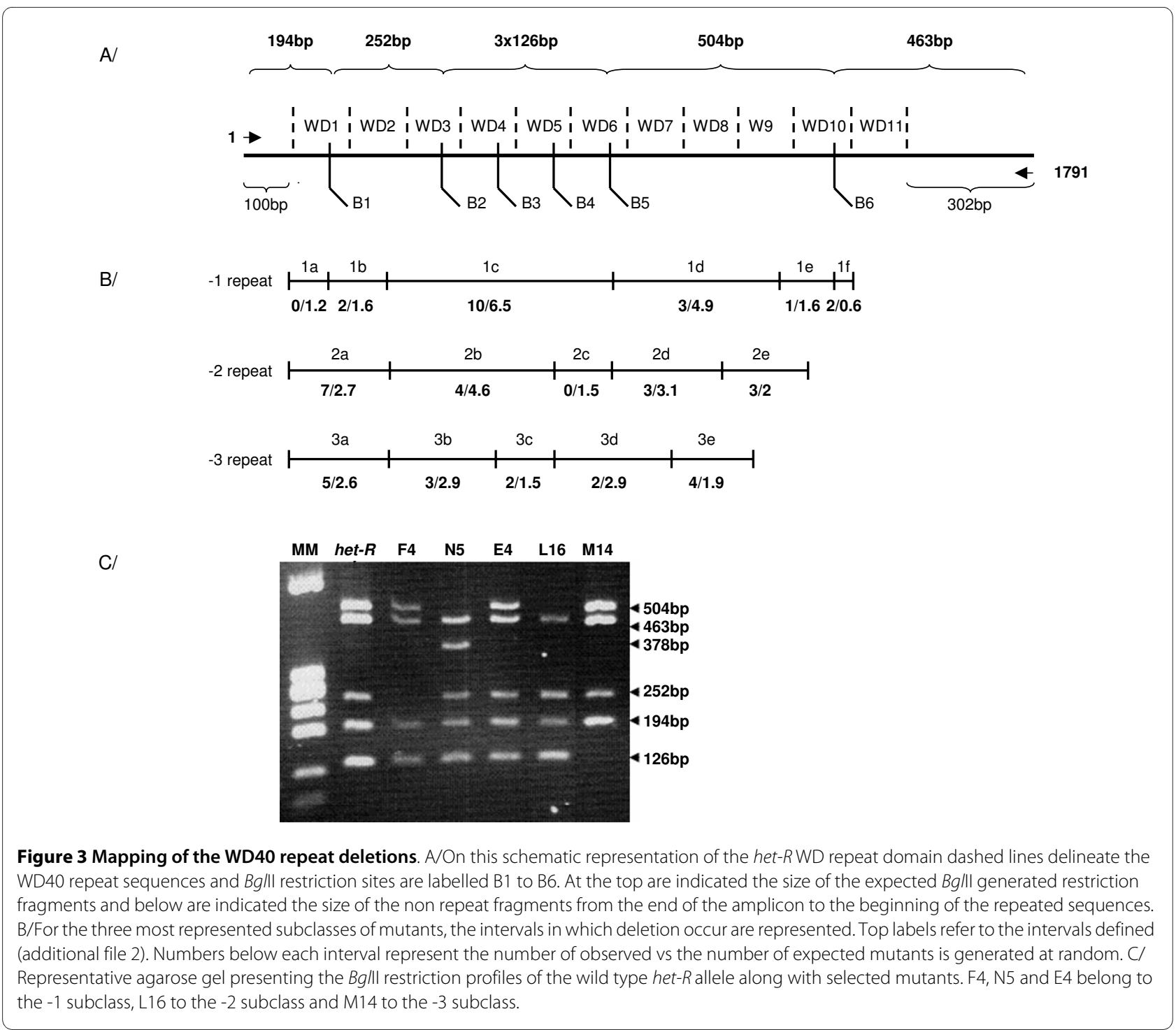

assuming that only deletions of contiguous WD40 repeats occurred, we predicted the intervals in which deletions could occur to generate the predicted BglII restriction profiles (additional file 2). The fact that 88 out of 95 mutants resulted in predicted BglII restriction profiles suggests that indeed deletions of a unique fragment of contiguous WD40 repeat sequences is the most frequent event. The 7 mutants not producing predicted $B g l I I$ restriction profiles probably resulted from more complex rearrangements. Finally for the 3 most frequent subclasses of mutants as defined by the number of deleted WD40 repeats, we compared the number of occurrence of deletions in each interval to $(E)$, the number of excepted deletions per interval if they occurred at random along the sequence. E depends on the length of the interval, the length of the WD repeat domain and the number of mutants in the subclass (Figure. 3 and additional file 2). This analysis demonstrated that deletions can involve any of the 11 repeats and that deletions occur at random along the WD40 repeat domain. We could not conduct the same analysis for the het-e mutants as BglII sites were present in 10 of the 11 WD40 repeats.

\section{Sequence of $\mathbf{2 5}$ mutant alleles}

To characterize the molecular events leading to inactivation of the het-R or het-E alleles in the different classes, we systematically sequenced the WD domains from the 21 individuals of the snWD and gWD classes of mutants as well as 4 alleles of the dWD class selected randomly. Five of the snWD mutants from the RV collection display a WD repeat domain identical to wild-type but displayed mutations outside of the WD-domain and will be described in a further section.

The remaining 16 alleles were affected in the WDrepeat domain (figure 4). Overall we found 38 new WD40 repeat sequences as compared to the parental alleles. 

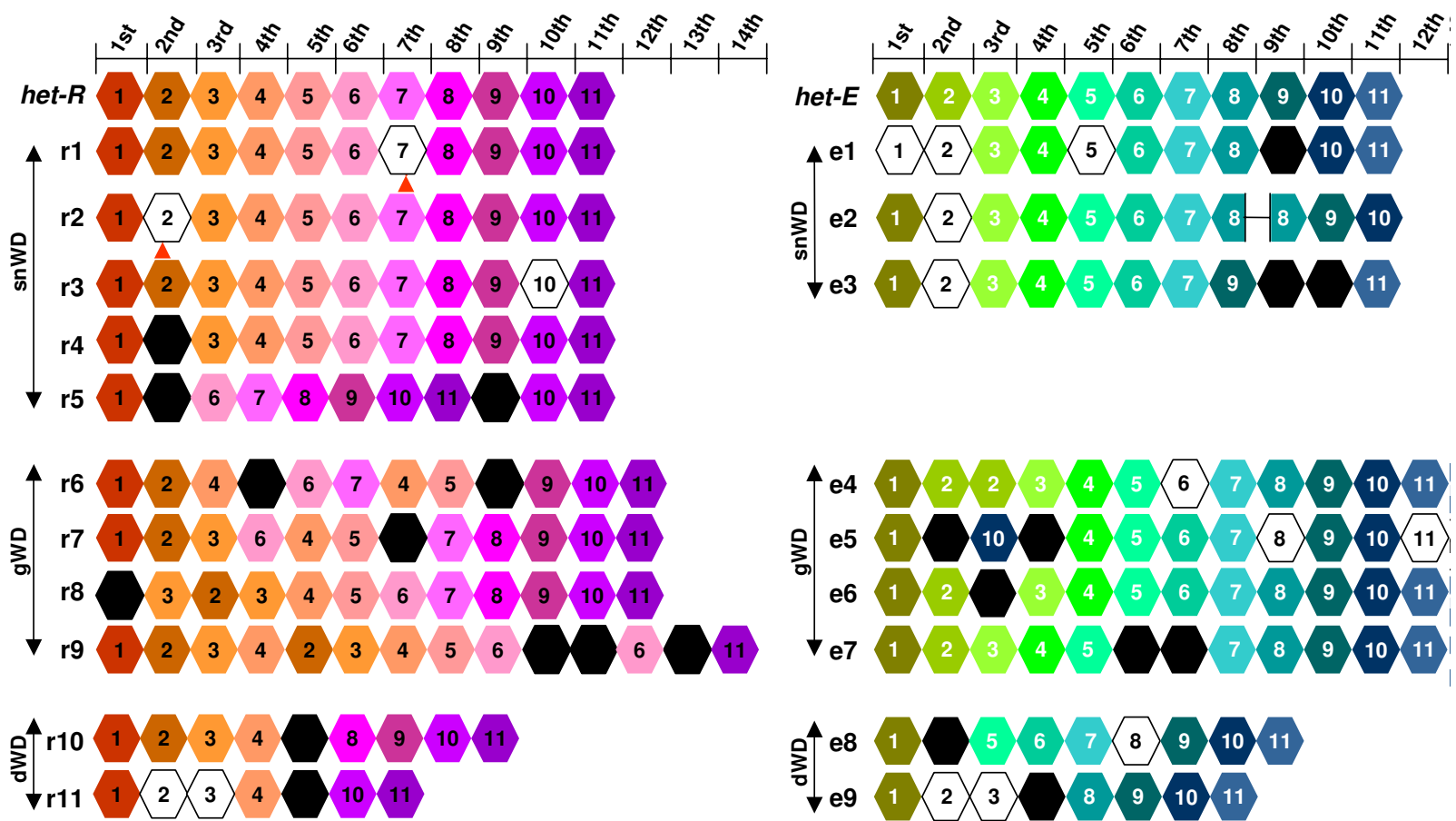

Figure 4 Schematic representation of the gWD and snWD mutants. A/Parental active het-R allele and mutants from the RV collection. B/Parental active het- $E$ allele and mutants from the CE collection. Nucleic sequence WD40 repeat units sharing the same colour are identical. Repeat units represented in white differ by a single nucleotide from their parental repeat unit (indicated by a number). Repeat units represented in black differ by more than one nucleotide from any parental units. Stop codons are indicated by arrowheads. Incomplete boxes of the e2 mutant delineate a 21 bp in frame deletion.

First mutations in the WD repeat domain all preserved the reading frame. In the simplest situation mutant alleles differed from wild type by a single point mutation in one of the WD-repeat units as exemplified by the $r 1$ and $r 2$ alleles mutated in repeat unit 7 and 2 respectively. Then, in the sequenced dWD alleles, a single deletion step led to the removal of 2, 3 or 4 contiguous repeat units in the $r 10$, $r 11, e 8$ or $e 9$ deletion mutants. Duplication of one or several repeat units is also observed like duplication of repeat unit 2 in the $e 4$ allele. Complex rearrangements occurred in several alleles with concomitant duplication, replacement and deletion events that imply multiple steps as exemplified by allele $r 9$ which displays a total of 14 repeat units.

\section{Repeat rearrangements are intragenic}

One of the striking features of the mutant allele sequences is that in the vast majority of the alleles, one finds new WD repeat units. We found 16 repeat units that differ from a parental unit by a single mutation and
22 repeat units that differ from the parental units by more than a single point mutation (figure 4). We observed 12 such new WD40 repeat unit sequences in the RV mutant collection and 10 in the CE mutant collection (figure 4). To define the origin of these 22 sequences, and because the WD40 repeat sequences of the hnwd gene family are undergoing concerted evolution and can thus occasionally be exchanged between loci, we sequenced the WD domain of all the family members from both parental strains used to produce the het- $R$ het- $V$ and het- $C$ het- $E$ SI strains. This lead to the identification of 41 new WD40 repeat units in addition to the 69 units already found in the sequenced genome [36]. A high level of polymorphism is evident at the $n w d$ loci between the two parental strains, both in terms of number of WD40 repeat units present at each locus and in terms of WD40 repeat unit sequences (additional file 3 ). As these strains are nearly isogenic [37], the observed polymorphism could reflect polymorphism existing in the natural isolates from which 
they are derived. Alternatively, this polymorphism could be the result of unselected concerted evolution occurring during crosses realised in laboratory conditions over the years as observed for rDNA loci in Drosophila melanogaster [38] or Daphnia obtus [39]. A phylogenetic tree was then constructed with the nucleotide sequences of the 157 WD40 repeat units from the wild-type alleles and including the 29 new WD40 repeat units identified in the mutant het-r and het-e alleles (additional file 4) that revealed a topology consistent with what we previously observed [18]. The WD40 repeat units tend to cluster by gene of origin with a strong bootstrap support. This indicates that intragenic events leading to sequence homogenization of WD40 repeat sequences are more frequent than intergenic exchanges. Significantly, all the newly identified WD40 repeat sequences found in the het-r mutant alleles cluster with the wild-type WD40 repeat sequences from the het- $R$ allele. Similarly, all newly identified WD40 repeat sequences at the het-e locus cluster with the WD40 repeat sequences from the parental het-E allele. We also analyzed the WD-repeat number of all $n w d$ gene family members in all mutant strains. None of the other $n w d$ genes showed any variation in repeat unit number in the mutants, consistent with the conclusion that rearrangements occurred at the intragenic level only (data not shown).

From these observations, we conclude that although intergenic WD40 repeat unit exchanges between $n w d$ loci apparently occur in the wild [18], in our experiment the new WD40 repeat sequences have been generated through intragenic events only.

\section{Rearrangements generate multiple novel repeat unit sequences}

As mentioned above in most of the mutant alleles one or several repeat units with novel sequences are found. One can attempt to recapitulate the events that led to the genesis of such novel repeat units. The simplest situation is probably the one that occurred in the alleles of the dWD class. Mutants $r 10$ and $r 11$ of the RV collection were deleted of 2 or 4 WD40 repeat units respectively. The deletion event resulted from fusions between repeats 5 and 7 and between repeats 5 and 9 of the wild-type allele in mutants $r 10$ and $r 11$ respectively. This fusion event generates new WD40 repeat units sequences. The same was true for the two dWD mutants of the CE collection (e8 and $e 9$ ). Again we found that in both cases a single fragment was deleted and resulted in new chimeric WD40 repeat sequences (additional file 5 ).

In mutant $r 4$ allele, 7 point mutations clustered in $88 \mathrm{bp}$ are observed in the $2^{\text {nd }}$ repeat of the domain. This stretch of 88 nucleotides appears almost identical to the corresponding region of the $4^{\text {th }}$ WD40 repeat sequence of the wild type allele and differs by only two positions located at the end of this region (additional file 6). This cluster of mutations could thus be the result of gene conversion of this short region in the mutant WD40 repeat sequence by the wild type sequence of the $4^{\text {th }}$ WD40 repeat unit.

A simple case of unequal crossing over could explain the genesis of the mutant allele e6. This mutant has gained a WD40 repeat unit e6-3 whose sequence is new. Comparison of the WD40 unit sequences reveals that this new repeat could correspond to a fusion between the $5^{\prime}$ end of the third repeat and the 3 'end of the second repeat of the wild type allele. The break point could have occurred at the adenine nucleotide at position 49 that is unique amongst the WD repeats (additional file 7).

We have previously shown that two pairs of codons (pairs of codons 7, 9 and 25, 27) in each repeat unit are under positive selection. Based on homology modelling, the corresponding amino acids all group at surface loops at the top of the $\beta$-propeller structure at the interaction surface [18]. The amino acid combination at those four positions thus presumably defines the functional binding properties of the repeat unit. In the 41 new WD40 repeat units we identified in the whole of the $n w d$ gene family, 23 displayed new amino acids and associations of amino acids at these four positions that had not been described in the genome sequence (additional file 8). More significantly, among the 38 new WD40 repeat units generated through mutations of the het- $E$ or het- $R$ alleles, twelve display novel amino acid combinations at those 4 critical positions that were not found in the repeat units of the parental alleles (table 1). Nine of these new combinations apparently arose by shuffling parental pairs of codons. However three mutant WD40 sequences most likely generated by point mutations lead to pairs of codons absent from the parental sequence. This observation strongly suggests that repeats with novel functional properties can indeed be generated through these rearrangement events as demonstrated in vitro for TPR or LRR containing repeat units $[40,41]$.

\section{Point mutations outside of the WD-repeat domain}

Five of the snWD mutants from the RV collection display a WD repeat domain identical to the wild type repeat domain. We then sequenced the remaining of these mutant genes after PCR amplification with primers $C$ and $\mathrm{D}$ (additional file 1). After comparisons with the wild type allele we identified one nucleotide insertion in mutants E5 and M8 and a one nucleotide deletion in mutant A1 that lead to frame shifts and premature stop codons (additional file 9). In the J4 mutant we identified a T66G substitution that results in an I22 M mutation (additional file 9). Interestingly this position belongs to the first of three conserved blocks forming the HET domain as defined earlier [19] and responsible for the induction of the cell death reaction [21]. In the M12 mutant a T1702A 
Table 1: Amino acid combinations at the protein protein interaction surface.

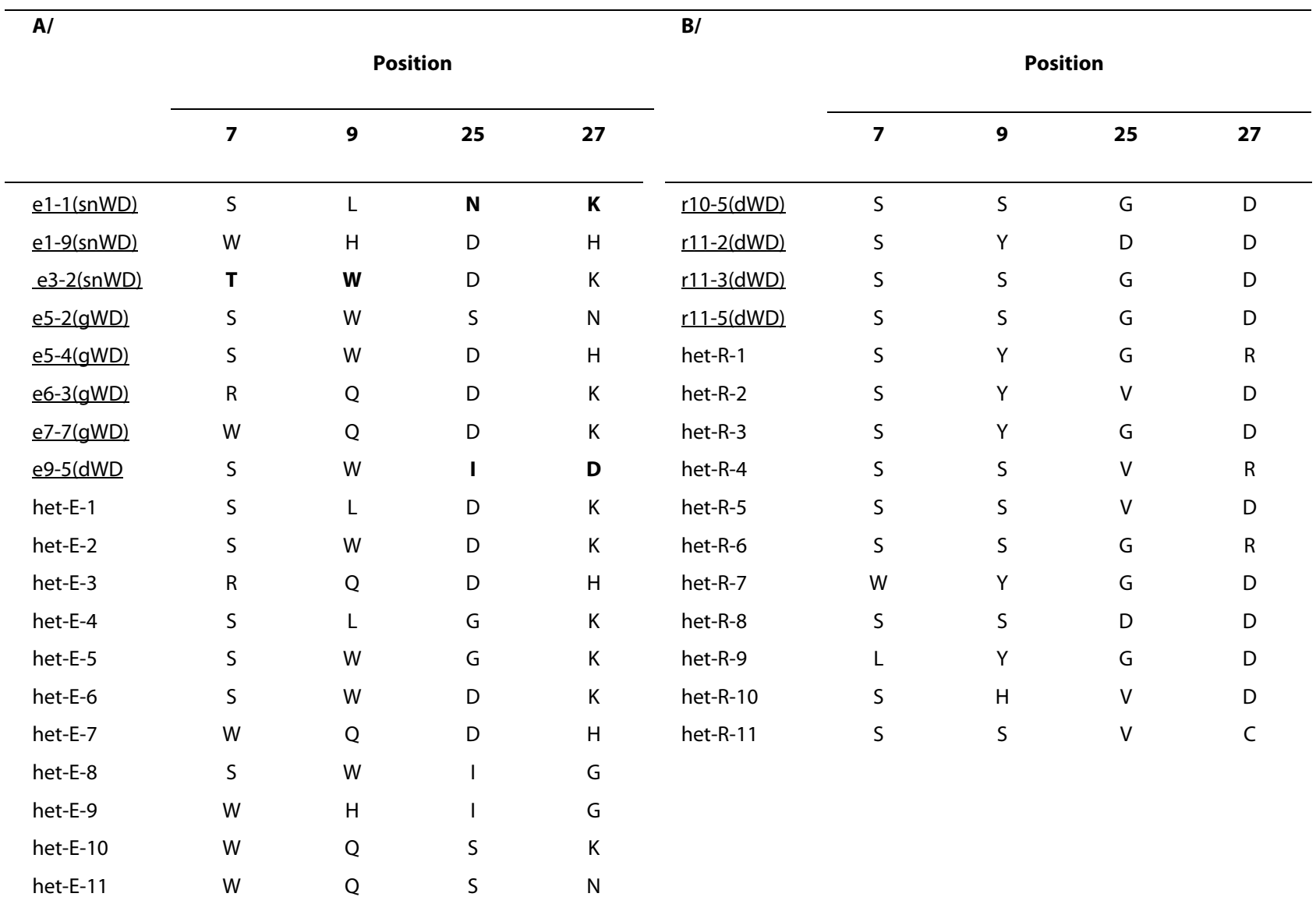

The four amino acids at positions under selection in newly generated alleles (underlined) are amino acids found in the parental sequences (white boxes) for $\mathrm{A} /$ the het- $E$ gene, and $\mathrm{B} /$ the het-R gene. Bolded pairs of amino acids were generated in the mutants. Repeats are named after their positions in the mutated alleles, the class of the corresponding mutant is indicated in brackets.

substitution leads to a Y568N mutation (additional file 9). This position is located between the conserved block $\mathrm{V}$ and VI of the NACHT domain [22] that is present in many different proteins of the STAND class [26]. The NACHT domain activity is essential to the incompatibility reaction [23].

\section{Discussion}

We have selected for mutations in two members of the $h n w d$ gene family that suppress self-incompatibility. In other words the incompatible genetic interaction between the HNWD genes and their antagonist het- $C$ or het- $V$ genes has been lost through mutation of the $H N W D$ partner. The vast majority of mutants were altered in their WD repeat domain involved in recognition specificity. The WD repeat domain adopts a doughnut shaped $\beta$ propeller fold that forms a protein-protein interaction surface at its top [42]. A typical $\beta$ propeller fold associates seven WD40 repeats, although such structures containing between 4 to 8 repeats have been described $[43,44]$. Proteins including larger number of WD40 repeat units have been described, and for example the thirteen WD40 repeat units of APAF-1 forms two distinct $\beta$ propeller structures of six and seven repeat units $[45,46]$. As we do not know how the WD repeat units of the HNWD proteins assemble, the reasons for the loss of activity in the mutants can only be speculated, but it is reasonable to suspect that loss of the genetic incompatibility in the mutants results from alterations of the physical interaction between the WD repeat domain of HET-R or HET-E proteins with their antagonist partner HET-V or HET-C. Most mutations correspond to deletions of WD40 repeat units. Large deletions leading to WD repeat domains encoding for less than 6-7 WD repeat units are unlikely to associate into a functional $\beta$ propeller thereby producing an inactive protein. In contrast small deletion mutants, snWD mutants and gWD mutants retain a number of repeats sufficient to generate a $\beta$-propeller fold. This suggests that the mutations specifically affect the binding affinity of the WD domain for the antagonist 
partner after the rearrangement of the amino acids located at the protein-protein interaction surface. The fact that in the RV collection repeat deletions that inactivate het-R occur all along the sequence of the repeat domain suggests that all the WD40 repeat units contribute to binding of the antagonist partner.

We obtained a total of 299 spontaneous mutants sectors starting from 153 colonies. All the mutants analysed are affected in the hnwd gene involved in the system. We found no mutants affected in the het-C or het- $V$ genes, or in extragenic suppressors. Also interestingly in a similar screen for mutants escaping from VI cell death with a genetically engineered het-s/het-S SI strain, Coustou and co-authors obtained only 17 mutants from 500 starting colonies after mutagenic treatment [47]. This difference in the mutant screening yields highlights the instability of the hnwd genes as opposed to the repeat-free het-s, het-S genes, het-c or $\bmod A$ genes [48]. In that respect it is relevant to note that the het- $E$ allele we used here displays an additional WD40 repeat unit compared to the allele originally described [25] while both recognize the same hetC1 allele. This difference is the likely consequence of genetic modification that occurred during subcultures in laboratory conditions.

A remarkable consequence of the instability of the WD repeat domain is that it contributes to the diversification of the WD40 repeat repertoire. Not only are repeat arrays reshuffled and specific repeats duplicated or deleted but numerous novel repeat unit sequences were generated in the process. In the subset of mutants we analysed we found a total of 38 new WD40 repeat units. More significantly, we found 12 new combinations of the four positions of the WD40 repeat unit located at the top of the $\beta$ propeller structure and thought to be crucial for recognition of the antagonistic partner [18]. We used here an experimental set up starting from a SI strain grown under permissive conditions and transferred to non permissive conditions to select for loss of function mutations as a consequence of the plasticity of the WD repeat domain of the hnwd gene family (figure 1). The genetic plasticity unveiled here might provide an explanation for a remarkable result obtained in early genetic studies on incompatibility interaction in P. anserina. Delettre and Bernet [49] have shown that using an ad hoc screen several additional non-allelic incompatibility loci could be genetically selected in laboratory conditions. The incompatibility interactions selected in that manner shared a number of features with the het-C/het-E and het-R/het- $V$ interactions, for instance they could be alleviated by the suppressor mutations that suppress het-C/het-E and het-R/ het- $V$ incompatibility. These new het loci also appeared to be genetically unstable. Based on the results presented herein, a plausible explanation for this result is that rearrangements in repeat arrays of hnwd genes generated novel binding specificities leading to recognition of a self determinant creating a genetic incompatibility de novo. Similarly, in nature, from the pool of WD40 repeats arrangements produced in the mycelium under vegetative growth conditions, selection will fish out combinations providing novel advantageous recognition specificities. It is relevant to note that het-c, the antagonistic locus of het- $d$ and het-e in VI, also displays signs of fast evolution [29] suggesting a co-evolution with the hnwd genes, thus new binding specificities might also be generated through evolution of het-c.

In biotechnological applications, it has been demonstrated in vitro that random association of different ankyrin repeat units can produce engineered ankyrin domains with binding affinity for selected targets [40,41], the same is true for designed LRR- and tetratricopeptiderepeat proteins. The combinatorial reshuffling and individual repeat generation process unveiled here suggest that the WD40 domains of $n w d$ genes might allow generation of a virtually unlimited binding repertoire. The generation of extensive binding repertoires by combinatorial association of variable intragenic tandem repeats is a strategy that is also encountered in other eukaryotic nonself recognition molecules in plants and animals. In plants recognition of pathogens is ensured by members of the NBS-LRR genes encoding for a NBS nucleotide binding site and a Leucine Rich Repeat domain. The LRR domain is thought to contribute to pathogen recognition [50,51]. Some $L R R$ genes are submitted to positive diversifying selection, concerted evolution and recombination between and within loci $[52,53]$. Evolution of the NBS$L R R$ genes appears enhanced in presence of the pathogen as presence of pathogen attack induces the Systemic Acquired Recombination [54] preferentially in the NBS $L R R$ encoding genes [55]. Similarly, in jawless vertebrates antigen recognition by lymphocyte receptors relies on the combinatorial assembly of LRR gene segments [56]. It has been proposed that somatic recombination in the variable LRR-units has the potential to generate a repertoire of $10^{14}$ different lymphocyte receptors. It appears that diversification of the fungal hnwd non-self recognition genes follows similar principles including diversification and combinatorial shuffling of repeated units forming a recognition plateform. The estimated WD-domain repertoire is colossal $\left(>10^{12}\right)$ even if one only considers the 54 different repeat units identified in the sequenced genome as the building blocks for a 7 repeat WD-domain repertoire. This likely represents an underestimation since additional repeat unit variants were found in a different isolate and novel repeat units can be generated through repeat instability. In addition, the $\beta$-propeller structures might be made of more than 7 repeats as exemplified by the 11 repeat WD-domains of wild-type het-e and het$v[48]$. 
In the two examples cited above, the plant NBS-LRR genes and the sea lamprey lymphocyte receptors, the repeat proteins are involved in pathogen-detection and the extraordinary binding versatility of these proteins is related to the diversity of the pathogen associated patterns that are recognized. Now how should the evolvability of the hnwd genes be understand? The possibility to generate such a vast variety of binding specificities does not make obvious sense in the context of vegetative incompatibility especially considering that in N. crassa, incompatibility alleles are very stable, in finite number (two or three alleles) and evolutionarily very ancient. The evolvability of the hnwd genes along with other features of the VI reaction recently lead us to suggest that the hnwd genes might be components of a fungal innate immune system with the function in response to pathogenic non-self [11]. In this context, as in the two examples mentioned above in plants and jawless vertebrates, the ability to produce a wide range of non-self recognition molecules would allow for the detection of a wide range of pathogens and counteract their immune-avoidance strategies. It is interesting to note that in some cases cell surface antigen in pathogens rely on tandem repeat instability for rapid adaptation so it appears that similar weapons are use by host and pathogens in their ongoing arms race.

\section{Conclusions}

The causes and the consequences of genetic incompatibilities currently raise a keen interest among evolutionary biologist because of their role in speciation processes [12,57-59]. Fungal incompatibility provides a genetically well defined example of such a genetic incompatibility. $h n w d$ genes based non-allelic incompatibility in $P$. anserina leads to various degrees of pre and post-zygotic isolation [60]. The present study documenting the genetic instability and plasticity of the hnwd genes as well as the hybrid necrosis phenomenon caused by NBS-LRR genes in $A$. thaliana $[12,13]$ illustrate that particular evolutionary regimens promoting extensive and rapid variation among isolates of the same species can favour the arising of genetic incompatibilities.

\section{Methods}

\section{Strains, incompatibility relationships and selection of mutants}

Conditions and media used for $P$. anserina have been reviewed recently [61]. The reference $P$. anserina isolate called $s$ harbours the het-r, het- $V$, het-c2 and het-e4 alleles at the het-r, het- $v$, het-c and het-e loci and is nearly isogenic to all other strains used in this work [37]. Because these loci are involved in non allelic incompatibility, it is possible to recover self incompatible (SI) strains in the progeny of appropriate crosses. SI strains display a clear phenotype characterised by a growth arrest soon after spore germination and a generalized cell death reaction. A cross between the $s$ strain and a strain bearing the het-R and het-V1 alleles at the het-r and het-v loci will produce SI het- $R$ het- $V$ F1 progeny. As incompatibility between het- $R$ and het- $V$ alleles is thermosensitive, these SI strains can grow as wild type at $32^{\circ} \mathrm{C}$, while transfer to $26^{\circ} \mathrm{C}$ triggers the incompatibility reaction [16]. A cross between the $s$ strain and a strain bearing a single incompatible allele at the het-e locus (het- $E_{a}$ ) produce a het-C het- $E$ SI strain. Incompatibility in these SI strains is partially suppressed by the addition of $6 \mathrm{~g} / \mathrm{l}$ of dihydrostreptomycin in the culture medium [62]. We selected and grew het- $R$ het- $V$ or het-C2 het-E SI strains in permissive conditions $\left(32^{\circ} \mathrm{C}\right.$ or dihydrostreptomycin containing medium) for $24 \mathrm{~h}$ until the mycelia reached $1 \mathrm{~cm}$ in diameter, before transfer to non permissive conditions $\left(26^{\circ} \mathrm{C}\right.$ or dihydrostreptomycin free medium). After 5 days of incubation we observed spontaneous mutant sectors arising. We collected a single mutant sector per starting colony to avoid analysing several times the same mutant. Vegetative incompatibility was assessed by barrage testing and sexual compatibility was assessed by spermatization as previously described [15].

\section{Nucleic acid manipulation and nomenclature}

Routine genomic DNA extractions were performed as described by [63], or using the Plant DNA extraction kit (Qiagen) when high quality DNA was necessary. PCR were performed using the Long Range Template Taq polymerase (Roche), according to the manufacturer recommendations. Time for DNA extension was adjusted according to the length of the fragment to amplify. The oligonucleotides used, as well as their location in the various loci analysed are listed in additional file 1. PCR products were gel purified and cloned in the XL TOPO cloning vector (Invitrogen). Sequencing was performed by the Genome express company. We systematically sequenced cloned WD40 sequences from at least two independent PCR reactions. Accession numbers are listed in additional file 10. Nucleic acids and protein sequences were analysed with the program MEGA3 [64]. PCR fragments were column purified (Qiagen Minelute kit) before restriction with BglII (Biolabs).

Gene and loci names are italicized while protein names are not. Active alleles are indicated with capitals while inactive alleles and loci are indicated with lower cases.

\section{Additional material}

Additional file 1 Details of the primers used.

Additional file $\mathbf{2}$ Mapping of the deletions occurring in the dWD class of mutants of the RV collection.

Additional file $\mathbf{3}$ Schematic representation of the parental WD repeat domains of the hnwd gene family members. 


\begin{abstract}
Additional file 4 Neighbor joining phylogenetic tree constructed with the nucleic sequences corresponding to WD40 repeat units from all members of the P. anserina nwd gene family, along with the new complex WD40 repeat unit sequences from the RV and CE collections.

Additional file 5 Protein sequences of WD repeat domains of randomly selected deletion mutants from the RV collection compared with WD repeat domain of the original het- $R$ allele ( $A$ and $B$ ) of from the CE collection with the original het- $E$ allele ( $C$ and D) were compared.

Additional file 6 Evidences for occurrence of gene conversion.

Additional file 7 Evidences for occurrence of unequal crossing overs. Additional file 8 Combinations of amino acids found at position 7, 925 and 27 of the WD40 repeat units in the hnwd gene family analysed here. Additional file $\mathbf{9}$ Nucleic and peptidic sequences of het- $R$ mutant alleles affected in the HET or NACHT domain.

Additional file $\mathbf{1 0}$ List of the accession numbers for the sequences used in this study.
\end{abstract}

\section{Authors' contributions}

DC and MP carried out the experimental work. CC, SJS and MP participated in the design of the study and drafted the manuscript. All authors read and approved the final manuscript.

\section{Acknowledgements}

We wish to thank Martine Sicault for invaluable technical assistance, Dr. F. Ness for stimulating discussions, and the EU (Transdeath grant \# 511983), the Agence Nationale pour la Recherche (ANR SexDevMycol \# NT-1_41707) and the Conseil Régional d'Aquitaine (\# 2005130009AB) for funding.

\section{Author Details}

Laboratoire de Génétique Moléculaire des Champignons, IBGC, UMR 5095 Université Victor Segalen Bordeaux2 et CNRS, 1 rue Camille Saint-Saëns,33077 Bordeaux Cedex, France

Received: 29 October 2009 Accepted: 6 May 2010 Published: 6 May 2010

\section{References}

1. Richman A: Evolution of balanced genetic polymorphism. Mol Ecol 2000, 9(12):1953-1963.

2. Drake JW, Charlesworth B, Charlesworth D, Crow JF: Rates of spontaneous mutation. Genetics 1998, 148(4):1667-1686.

3. Aanen DK, Debets AJ, de Visser JA, Hoekstra RF: The social evolution of somatic fusion. Bioessays 2008, 30(11-12):1193-1203.

4. Saupe SJ: Molecular genetics of heterokaryon incompatibility in filamentous ascomycetes. Microbiol Mol Biol Rev 2000, 64(3):489-502.

5. Glass NL, Dementhon K: Non-self recognition and programmed cell death in filamentous fungi. Curr Opin Microbio/ 2006, 9(6):553-558.

6. Pinan-Lucarre B, Paoletti M, Clave C: Cell death by incompatibility in the fungus Podospora. Semin Cancer Biol 2007, 17(2):101-111.

7. Milgroom MG, Cortesi P: Biological control of chestnut blight with hypovirulence: a critical analysis. Annu Rev Phytopathol 2004 42:311-338

8. Biella S, Smith ML, Aist JR, Cortesi P, Milgroom MG: Programmed cell death correlates with virus transmission in a filamentous fungus. Proc Biol Sci 2002, 269(1506):2269-2276.

9. Debets AJM, Griffiths AJF: Polymorphism of het-genes prevents resource plundering in Neurospora crassa. Mycol Res 1998, 102(11):1343-1349.

10. Aanen DK, Debets AJ, de Visser JA, Hoekstra RF: The social evolution of somatic fusion. Bioessays 2008, 30(11-12):1193-1203.

11. Paoletti M, Saupe SJ: Fungal incompatibility: Evolution origin in pathogen defense? Bioessays 2009 in press.

12. Bomblies K, Weigel D: Hybrid necrosis: autoimmunity as a potential gene-flow barrier in plant species. Nat Rev Genet 2007, 8(5):382-393.

13. Bomblies K, Lempe J, Epple P, Warthmann N, Lanz C, Dangl JL, Weigel D: Autoimmune response as a mechanism for a Dobzhansky-Muller-type incompatibility syndrome in plants. PLoS Bio/ 2007, 5(9):e236.

14. Saupe SJ, Clave C, Begueret J: Vegetative incompatibility in filamentous fungi: Podospora and Neurospora provide some clues. Curr Opin Microbiol 2000, 3(6):608-612.
15. Chevanne D, Bastiaans E, Debets A, Saupe SJ, Clave C, Paoletti M: Identification of the het-r vegetative incompatibility gene of Podospora anserina as a member of the fast evolving HNWD gene family. Curr Genet 2009, 55(1):93-102.

16. Labarere J: [Properties of an incompatibility system in Podospora anserina fungus and value of this system for the study of incompatibility]. CR Acad Sci Hebd Seances Acad Sci D 1973 276(8):1301-1304

17. Espagne E, Lespinet O, Malagnac F, Da Silva C, Jaillon O, Porcel BM Couloux A, Aury JM, Ségurens B, Poulain J, et al:: The genome sequence of the model ascomycete fungus Podospora anserina. Genome biology 2008, 9(5):R77.

18. Paoletti $M$, Saupe SJ, Clavé C: Genesis of a fungal non self recognition repertoire. PLOS ONE 2, e283 2007

19. Smith ML, Micali OC, Hubbard SP, Mir-Rashed N, Jacobson DJ, Glass NL: Vegetative incompatibility in the het- 6 region of Neurospora crassa is mediated by two linked genes. Genetics 2000, 155(3):1095-1104.

20. Kaneko I, Dementhon K, Xiang Q, Glass NL: Nonallelic interactions between het-c and a polymorphic locus, pin-c, are essential for nonself recognition and programmed cell death in Neurospora crassa. Genetics 2006, 172(3):1545-1555.

21. Paoletti $M$, Clave $C$ : The fungus-specific HET domain mediates programmed cell death in Podospora anserina. Eukaryot Cell 2007, 6(11):2001-2008.

22. Koonin EV, Aravind L: The NACHT family - a new group of predicted NTPases implicated in apoptosis and MHC transcription activation. Trends Biochem Sci 2000, 25(5):223-224

23. Espagne E, Balhadere $P$, Begueret J, Turcq B: Reactivity in vegetative incompatibility of the HET-E protein of the fungus Podospora anserina is dependent on GTP-binding activity and a WD40 repeated domain. Mol Gen Genet 1997, 256(6):620-627.

24. Espagne E, Balhadere P, Penin ML, Barreau C, Turcq B: HET-E and HET-D belong to a new subfamily of WD40 proteins involved in vegetative incompatibility specificity in the fungus Podospora anserina. Genetics 2002, 161(1):71-81.

25. Saupe S, Turcq B, Begueret J: A gene responsible for vegetative incompatibility in the fungus Podospora anserina encodes a protein with a GTP-binding motif and G beta homologous domain. Gene 1995, 162(1):135-139.

26. Leipe DD, Koonin EV, Aravind L: STAND, a class of P-loop NTPases including animal and plant regulators of programmed cell death: multiple, complex domain architectures, unusual phyletic patterns, and evolution by horizontal gene transfer. J Mol Biol 2004, 343(1):1-28.

27. Danot O, Marquenet E, Vidal-Ingigliardi D, Richet E: Wheel of Life, Wheel of Death: A Mechanistic Insight into Signaling by STAND Proteins. Structure 2009, 17(2):172-182.

28. Galagan JE, Selker EU: RIP: the evolutionary cost of genome defense. Trends Genet 2004, 20(9):417-423.

29. Saupe S, Turcq B, Begueret J: Sequence diversity and unusual variability at the het-c locus involved in vegetative incompatibility in the fungus Podospora anserina. Curr Genet 1995, 27(5):466-471.

30. Paoletti M, Saupe SJ: Fungal incompatibility: evolutionary origin in pathogen defense? Bioessays 2009, 31(11):1201-1210.

31. Armour JA: Tandemly repeated DNA: why should anyone care? Mutat Res 2006, 598(1-2):6-14

32. Richard GF, Kerrest A, Dujon B: Comparative genomics and molecular dynamics of DNA repeats in eukaryotes. Microbio/ Mol Biol Rev 2008, 72(4):686-727.

33. Verstrepen KJ, Jansen A, Lewitter F, Fink GR: Intragenic tandem repeats generate functional variability. Nat Genet 2005, 37(9):986-990.

34. Gibbons JG, Rokas A: Comparative and functional characterization of intragenic tandem repeats in 10 Aspergillus genomes. Mol Biol Evol 2009, 26(3):591-602

35. Fondon JW, Garner HR: Molecular origins of rapid and continuous morphological evolution. Proc Natl Acad Sci USA 2004, 101(52):18058-18063

36. Paoletti M, Saupe SJ, Clave C: Genesis of a fungal non-self recognition repertoire. PLOS ONE 2007, 2(3):e283.

37. Bernet J: Mode d'action des gènes de "barrage" et relation entre l'incompatibilité cellulaire et l'incompatibilité sexuelle chez Podospora anserina. Annales des sciences naturelles, botanique 1965, 12(VI):611-768. 
38. Averbeck KT, Eickbush TH: Monitoring the mode and tempo of concerted evolution in the Drosophila melanogaster rDNA locus. Genetics 2005, 171(4):1837-1846.

39. MCTaggart SJ, Dudycha JL, Omilian A, Crease TJ: Rates of recombination in the ribosomal DNA of apomictically propagated Daphnia obtusa lines. Genetics 2007, 175(1):311-320.

40. Binz HK, Amstutz P, Pluckthun A: Engineering novel binding proteins from nonimmunoglobulin domains. Nat Biotechnol 2005, 23(10):1257-1268

41. Binz HK, Amstutz P, Kohl A, Stumpp MT, Briand C, Forrer P, Grutter MG, Pluckthun A: High-affinity binders selected from designed ankyrin repeat protein libraries. Nat Biotechnol 2004, 22(5):575-582.

42. Sprague ER, Redd MJ, Johnson AD, Wolberger C: Structure of the Cterminal domain of Tup1, a corepressor of transcription in yeast. Embo J 2000, 19(12):3016-3027.

43. Smith TF, Gaitatzes C, Saxena K, Neer EJ: The WD repeat: a common architecture for diverse functions. Trends Biochem Sci 1999, 24(5):181-185.

44. Paoli M: Protein folds propelled by diversity. Prog Biophys Mol Biol 2001, 76(1-2):103-130

45. Yu X, Wang L, Acehan D, Wang X, Akey CW: Three-dimensional structure of a double apoptosome formed by the Drosophila Apaf-1 related killer. J Mol Biol 2006, 355(3):577-589.

46. Yu X, Acehan D, Menetret JF, Booth CR, Ludtke SJ, RiedI SJ, Shi Y, Wang X, Akey CW: A structure of the human apoptosome at 12.8 A resolution provides insights into this cell death platform. Structure 2005 , 13(11):1725-1735

47. Coustou V, Deleu C, Saupe SJ, Begueret J: Mutational analysis of the [Hets] prion analog of Podospora anserina. A short N-terminal peptide allows prion propagation. Genetics 1999, 153(4):1629-1640.

48. Barreau C, Iskandar M, Loubradou G, Levallois V, Begueret J: The mod-A suppressor of nonallelic heterokaryon incompatibility in Podospora anserina encodes a proline-rich polypeptide involved in female organ formation. Genetics 1998, 149(2):915-926.

49. Delettre YM, Bernet J: Regulation of proteolytic enzymes in Podospora anserina: selection and properties of self-lysing mutant strains. Mol Gen Genet 1976, 144(2):191-197.

50. McDowell JM, Simon SA: Molecular diversity at the plant-pathogen interface. Dev Comp Immunol 2007.

51. Friedman AR, Baker BJ: The evolution of resistance genes in multiprotein plant resistance systems. Curr Opin Genet Dev 2007, 17(6):493-499.

52. Mondragon-Palomino M, Gaut BS: Gene conversion and the evolution of three leucine-rich repeat gene families in Arabidopsis thaliana. Mol Biol Evol 2005, 22(12):2444-2456.

53. Nagy ED, Bennetzen JL: Pathogen corruption and site-directed recombination at a plant disease resistance gene cluster. Genome Res 2008, 18(12):1918-23.

54. Molinier J, Ries G, Zipfel C, Hohn B: Transgeneration memory of stress in plants. Nature 2006, 442(7106):1046-1049.

55. Boyko A, Kathiria P, Zemp FJ, Yao Y, Pogribny I, Kovalchuk I: Transgenerational changes in the genome stability and methylation in pathogen-infected plants: (virus-induced plant genome instability). Nucleic Acids Res 2007, 35(5):1714-1725.

56. Alder MN, Rogozin IB, Iyer LM, Glazko GV, Cooper MD, Pancer Z: Diversity and function of adaptive immune receptors in a jawless vertebrate. Science 2005, 310(5756):1970-1973.

57. Schartl M: Evolution of Xmrk: an oncogene, but also a speciation gene? Bioessays 2008, 30(9):822-832.

58. Greig D: Reproductive isolation in Saccharomyces. Heredity 2009, 102(1):39-44

59. Presgraves DC: Speciation genetics: epistasis, conflict and the origin of species. Curr Biol 2007, 17(4):R125-127.

60. Durrens $P$, Laigret F, Labarere J, Bernet J: Podospora anserina mutant defective in protoperithecium formation, ascospore germination, and cell regeneration. J Bacterio/ 1979, 140(3):835-842.

61. Pinan-Lucarre B, Clave C: Monitoring autophagy in the filamentous fungus Podospora anserina. Methods Enzymol 2008, 451:251-270.

62. Bernet J, Begueret J, Labarere J: Incompatibility in the fungus Podospora anserina. Are the mutations abolishing the incompatibility reaction ribosomal mutations? Mol Gen Genet 1973, 124(1):35-50.
63. Lecellier G, Silar P: Rapid methods for nucleic acids extraction from Petri dish-grown mycelia. Curr Genet 1994, 25(2):122-123.

64. Kumar S, Tamura K, Nei M: MEGA3: Integrated software for Molecular Evolutionary Genetics Analysis and sequence alignment. Brief Bioinform 2004, 5(2):150-163.

doi: $10.1186 / 1471-2148-10-134$

Cite this article as: Chevanne et al. WD-repeat instability and diversification of the Podospora anserina hnwd non-self recognition gene family BMC Evolutionary Biology 2010, 10:134

\section{Submit your next manuscript to BioMed Central} and take full advantage of:

- Convenient online submission

- Thorough peer review

- No space constraints or color figure charges

- Immediate publication on acceptance

- Inclusion in PubMed, CAS, Scopus and Google Scholar

- Research which is freely available for redistribution 